\title{
The atomistic mechanism of fast relaxation processes in $\mathrm{Cu}_{65} \mathrm{Zr}_{35}$ glass
}

\author{
P. Palomino Rico ${ }^{\text {a }}$, D.G. Papageorgiou ${ }^{b}$, A.L. Greer ${ }^{c}$, G.A. Evangelakis ${ }^{a}$
}

a) University of Ioannina, Department of Physics, Ioannina 45110, Greece

b) University of Ioannina, Department of Materials Science and Engineering, Ioannina 45110, Greece

c) University of Cambridge, Department of Materials Science \& Metallurgy, Cambridge, CB3 OFS, U.K.

\begin{abstract}
Molecular-dynamics simulations of the glass $\mathrm{Cu}_{65} \mathrm{Zr}_{35}$ show a rattling mode of atomic motion. While the frequency of the rattling mechanism is essentially independent of temperature and of the strain applied to the glass, the fraction of atoms undergoing rattling is dependent on temperature and strain. It is argued that the rattling motions constitute the 'fast processes' that lie between $\beta$ relaxation and the boson peak in the characteristic spectrum of dynamic modes in glasses. It is concluded that the fast processes are a precursor to $\beta$ relaxation and that their inhomogeneous distribution in the metallic glass is the origin of the shear transformation zones governing the onset of plastic flow.
\end{abstract}

Keywords: Amorphous alloy, Dynamic modes, Metallic glass, Molecular dynamics, Structural relaxation 


\section{Introduction}

Recent years have seen intense interest in fast dynamic processes in supercooled liquids and glasses [1,2]. For electrically insulating glasses, dielectric spectroscopy reveals a range of phenomena, intrinsic to the glassy state, spread over a wide range of frequencies (Fig. 1). The $\alpha$ relaxation $\left(10^{-6}-1 \mathrm{~Hz}\right)$ is associated with the glass transition; the $\beta$ or JohariGoldstein relaxation $\left(10^{-2}-10^{7} \mathrm{~Hz}\right)$ is associated with modes in loosely packed regions; the boson peak $\left(10^{12} \mathrm{~Hz}\right)$ is associated with an excess in the low-temperature vibrational density of states; and infra-red bands are found at even higher frequency [1-3]. Lunkenheimer et al. [1] found that there are significant dynamic modes between the $\beta$ relaxation and the boson peak; in their comprehensive review of glass dynamics, they considered that the discovery of these intermediate fast processes $\left(10^{8}-10^{11} \mathrm{~Hz}\right)$ was "the most important outcome" of their extended dielectric investigations.

\section{Figure 1 near here}

In the present paper, we study such fast processes in a metallic glass. It is now understood that $\beta$ relaxation in metallic glasses is closely related to deformation mechanisms, the glass transition, atomic diffusion and the breakdown in the Stokes-Einstein relation, and to crystallization $[2,4,5]$. The fast processes have particular importance because they may be the threshold to $\beta$ relaxation. In particular, the fast processes may be critical in understanding the onset of plasticity in metallic glasses, and the link between that onset and structural inhomogeneity [6]. The fast processes are stronger relative to the boson peak for glasses formed from more fragile liquids [7]. Taking the intermediate-to-high fragility of metallic glass-forming liquids into account, fast processes may therefore be significant for metallic 
glasses. Understanding these processes and the onset of $\beta$ relaxation may assist in the design of glasses to optimize properties.

Dielectric spectroscopy is not applicable to metallic glasses, which have been investigated using techniques such as dynamic mechanical analysis (DMA), in which characteristic dynamic modes are revealed by peaks in the loss modulus as a function of temperature. There is an $\alpha$-relaxation peak, and $\beta$ relaxation is indicated by anything from an excess wing on the $\alpha$ peak, to a shoulder, to a distinct $\beta$ peak [5,8-10]. Recent studies have also found a second fast $\beta^{\prime}$ peak [11,12] and a near-constant-loss mode [13] on the lowtemperature side of the $\beta$ peak. The shifts of loss peaks to higher temperature at higher frequency give the effective activation energy of the modes. For $\beta$ relaxation in metallic glasses, the activation energy is $26( \pm 2) R T_{\mathrm{g}}[2,5]$; for the fast $\beta^{\prime}$ and near-constant-loss modes, it is roughly half this value [11-13]. In the context of the present work, we note that binary $\mathrm{Cu}-\mathrm{Zr}$ glasses exhibit $\beta$ relaxation as a shoulder on the $\alpha$ peak [9].

DMA has a limited frequency range, and is incapable of revealing the boson peak. This has, however, been detected in measurements of low-temperature specific heat of CuZr-based metallic glasses [14], and the boson peak and $\beta$ relaxation are similarly affected by changes in metallic-glass composition [15]. In the absence of direct high-frequency spectroscopy measurements, molecular dynamics (MD) has proved to be a useful tool in elucidating structural and dynamic aspects of the boson peak. Jakse et al. [16] used MD to simulate a $\mathrm{Cu}_{50} \mathrm{Zr}_{50}$ (at.\%) glass and found a well-defined peak consistent with the specific-heat measurements [14]. They found that the boson peak is associated with the "rattling" of both species of atoms in localized regions of relatively low density and defective structure. The participating atoms vibrate with mean-square amplitudes that are abnormally large and also span a wide range, reflecting the diversity of local coordinations. For the boson peak similarly found in MD simulations of a $\mathrm{Cu}_{64} \mathrm{Zr}_{36}$ glass, Ding et al. [17] focused on the $1 \%$ of 
vibrational modes of lowest frequency. These soft modes lie in the region of interest for the fast processes in Fig. 1, but the time dependence of atomic positions was not reported.

In the present work, our MD simulations of a similar $\mathrm{Cu}-\mathrm{Zr}$ glass reveal additional modes that we identify with the fast processes described by Lunkenheimer et al. [1]. In contrast to the main relaxation peaks in Fig. 1, the boson peak is temperature-independent. The fast processes therefore sit in an interesting region between vibration (boson) and relaxation $\left(\beta\right.$ and $\left.\beta^{\prime}\right)$ behavior $[1,18]$.

\section{Methods}

For the present work, the glass $\mathrm{Cu}_{65} \mathrm{Zr}_{35}$ (at.\%) was chosen because of extensive prior studies of this system $[19,20]$. MD simulations of the glass were based on an embeddedatom-method potential [21] that has been proven to reproduce satisfactorily the basic properties of $\mathrm{Cu}-\mathrm{Zr}$ alloys, including metallic glasses. A system consisting of 31,250 atoms with periodic boundary conditions is initially melted at $2000 \mathrm{~K}$ and then cooled to $300 \mathrm{~K}$ at a rate of $2 \times 10^{12} \mathrm{~K} \mathrm{~s}^{-1}$. The simulations are performed at $300 \mathrm{~K}$ (with some at higher temperatures) with a time-step of $2 \mathrm{fs}$, in the isobaric-isothermal canonical ensemble with Nosé [22] and Andersen [23] daemons. Finally, equilibration for $2.2 \mathrm{~ns}$ in the microcanonical ensemble is used to ensure sufficient relaxation. For the tensile deformation we use a strain rate of $10^{8} \mathrm{~s}^{-1}$ up to a strain of $10 \%$, while in the lateral dimensions we impose strains corresponding to a Poisson ratio of 0.34 [24].

For the analysis of atomic coordination polyhedra, we use a procedure based on nonlinear multi-dimensional minimization [25] of prototype clusters, taking each atom of the system as the potential center of a cluster. Based on previous studies [19,20] we consider 
cluster geometries that were found to have significant populations in the $\mathrm{Cu}_{65} \mathrm{Zr}_{35}$ metallic glass: icosahedral (ICO), rhombic dodecahedral (RD) and cuboctahedral (CUB). Because it is easier to obtain data not only on perfect but also on distorted and/or truncated clusters, this classification is more useful than that based on Voronoi polyhedra [26], which, however, we also used for comparison.

\section{Results}

The structural analysis reproduced the results reported in many previous studies (for example in $[17,26])$, that the atomic coordination is based on interconnected ICO-like clusters, most of which are distorted. Our present interest, however, is mainly in the detailed characterization of the time dependence of atomic positions. Figure $2 \mathrm{a}$ shows the key finding, that for some atoms there is a clear alternation between two positions in each of which there is vibrational motion. The time dependence of atomic position seen in Fig. $2 \mathrm{a}$ is quite distinct from that found by Jakse et al. [16] (Fig. 3 in their paper) over very similar timescales and at the same temperature $(300 \mathrm{~K})$.

\section{Figure 2 near here}

We determined the atomic positions from the locations of their Gaussian-like time distributions, thus avoiding the influence of thermal vibration. In contrast with a crystal, the metallic glass does not have a lattice with well-defined equilibrium positions. As a consequence, the rattling between two sites shows a wide distribution of distances (from 0.1 to more than $1.5 \AA$ ) and life-times (from 1.5 to more than $100 \mathrm{ps}$ ). This distribution is wider 
than the range of amplitude ( 0.3 to $0.6 \AA$ ) found by Jakse et al. [16] to contribute to the boson peak. As is emphasized in Fig. 2a, the motion is to-and-fro; consequently the net mass flow is practically negligible (i.e., as expected, no diffusion is found at $300 \mathrm{~K}$ ). The rattling process revealed in Fig. $2 \mathrm{a}$ is shown with better time resolution in Fig. 2b. The alternation in atomic position is accompanied by changes in local coordination: the polyhedron around the rattling atom alternates between ICO and RD or CUB states as the atom moves to and fro.

It is remarkable that such rattling motions (with some amplitudes exceeding $1.5 \AA$ ) can occur, given that metallic glasses are so densely packed. There are no vacancies in metallic glasses, and their structure is considered to consist of efficiently packed clusters [21]. Our MD simulations do, however, reveal how such large-amplitude rattling is possible. Figure 3 shows atomic sites (with vibration eliminated) in a local region. This shows that two or three neighboring atoms can perform concerted motions to accommodate large-amplitude rattling. In state 1 (Fig. 3a), atoms $\mathrm{A}$ and $\mathrm{B}$, separated by $3.8 \AA$, are the central atoms in two touching (i.e. face-sharing) polyhedral clusters. In the rattling, atoms B, D, A and C have coordinated displacements of about $1 \AA$, comprising a string-like movement. Atom F is displayed in white because it was a neighbor of atom B in state 1 , but it is not its neighbor in state 2 , after atom $\mathrm{B}$ rattles to its other site. In the next stage (Fig. 3b), atom A does not move, while atom B returns to its initial location, and atom $\mathrm{F}$ becomes again part of its first-neighbor coordination shell. In this transition, five more atoms adjust their positions through small movements of the order of $0.3 \AA$ A. Finally (Fig. 3c), atom A returns near its initial location, followed by atom $\mathrm{C}$. The movement is facilitated by atom $\mathrm{D}$ that moves downwards, followed by atom B. Again, a string-like movement can be identified. The atoms A and B are now first neighbors, at a distance of $2.7 \AA$, their two clusters are interpenetrating (i.e. the central atom of one is in the first-neighbor shell of the other) and atom F is not anymore a 
nearest neighbor of atom $\mathrm{B}$, showing how the local structure of nearest-neighbor polyhedra has changed through the coordinated rattling motion.

\section{Figure 3 near here}

To characterize the extent of the rattling throughout the system, we counted the total number of events, considering one event as the alternation from one site to another and back. Imposing a minimum distance of $0.6 \AA$ and a minimum residence time of $1.5 \mathrm{ps}$, we calculated the frequency of occurrence of rattling, normalizing the number of events with respect to the number of atoms in the system. The result is a frequency of occurrence of 435 $\mathrm{MHz}$ at $300 \mathrm{~K}$, being in the transition region between $\beta$-relaxation and the fast processes in the Lunkenheimer plot [1]. In preliminary studies at higher temperatures, up to $600 \mathrm{~K}$ (still below $T_{\mathrm{g}}$ ), we find that this frequency increases by almost one order of magnitude.

Although atoms performing rattling movements come back to their initial positions, showing reversibility, their neighboring atoms may change positions, thus inducing local structural alterations. To quantify this, we performed an analysis of all the atoms involved in rattling in the quiescent glass (i.e. not subject to any external load). About $8 \%$ of all events induced irreversible changes in the neighborhood of the moving atoms, thus resulting in persistent local structural changes (Fig. 3c).

\section{Figure 4 near here}

Noting that the boson peak is associated with more loosely packed regions [16], we examine whether the rattling atoms in the present work show a similar association. Taking atomic radii of $1.28 \AA$ for $\mathrm{Cu}$ and $1.60 \AA$ for $\mathrm{Zr}$, a Voronoi tessellation was performed, and 
thereby the volume around each atom was defined. In our simulations, the atomic volume around the rattling atoms is larger (by $1 \%$ for $\mathrm{Cu}$ and $1.5 \%$ for $\mathrm{Zr}$ ) than the average atomic volume in the system (Fig. 4). Of all the atoms in the system, only $1.1 \%$ perform rattling motions, $1 \%$ of them being $\mathrm{Cu}$ and $0.1 \% \mathrm{Zr}$. The dominant role for the smaller atomic species is consistent with the link proposed [4] between the $\beta$-relaxation mode in metallic glasses and self-diffusion of the smallest constituent atoms.

Ding et al. [17] characterized the coordination polyhedra in their simulated $\mathrm{Cu}_{64} \mathrm{Zr}_{36}$ glass, distinguishing well-defined motifs (for example the full icosahedron with Voronoi index $\langle 0,0,12,0\rangle$ ) from geometrically unfavored motifs (GUMs). Atoms participating more in soft modes are more likely to be at the center of GUMs [17]. In the present work, classifying coordination polyhedra in the same way [17], we find that the fraction of $\mathrm{Cu}$ atoms at the center of GUMs is $65 \%$ overall, but is $80 \%$ for those participating in rattling. The equivalent fractions for the $\mathrm{Zr}$ atoms are $80 \%$ and $90 \%$. Thus, the rattling atoms are almost exclusively at the centers of GUMs, an even stronger association than that between soft modes and GUMs [17].

\section{Figure 5 near here}

The rattling atoms are not homogeneously distributed. Instead, they are gathered together, forming regions which vary widely in size and number of atoms (Fig. 5a). Given that rattling is concentrated in specific regions, it is interesting to consider the frequency in terms of only the moving atoms; this is distinct from the frequency of occurrence noted earlier, which was normalized with respect to all the atoms in the system. Normalizing with respect to the rattling atoms only, we obtain the frequency of the mechanism, which at $300 \mathrm{~K}$ has a value of the order of $40 \mathrm{GHz}$, thus falling exactly where fast processes are expected [1]. 
In contrast to the frequency of occurrence, this frequency shows little or no dependence on temperature ( $2 \%$ increase from $300 \mathrm{~K}$ to $600 \mathrm{~K})$. String-like concerted motion of atoms was noted in Fig. 3.

Given that the fast processes are intrinsic to the glassy state [1] it is of interest to explore their behavior when the glass is subjected to mechanical loading. We performed simulations under an imposed tensile strain of up to $10 \%$. Holding the strain at $10 \%$, we analyzed the rattling motions, and found a $45 \%$ increase in the number of events,

corresponding to a frequency of occurrence of $630 \mathrm{MHz}$. Clearly this frequency is influenced by the deformation. The number of atoms performing rattling motions also increases, reaching almost $2 \%$ of the atoms in the system, clustered together in larger regions (Fig. $5 \mathrm{~b}$ ). Taking the rattling atoms into account when calculating the frequency of the mechanism, we found that it remains at $40 \mathrm{GHz}$, suggesting weak or no dependence on the applied strain.

\section{Figure 6 near here}

The persistent structural change associated with the rattling motions becomes more evident as the straining proceeds. There is a systematic shift in the relative populations of different coordination polyhedra, with continuous transformation of the clusters from ICO to RD or CUB (Fig. 6a). The population of ICO clusters, which represent $43.4 \%$ of the total clusters in the system, is reduced to $41.6 \%$ (an absolute reduction of 1.8\%). Similar changes were found in earlier work on $\mathrm{Cu}_{46} \mathrm{Zr}_{54}$ glass [28], but the present work extends to much higher strains. Error bars represent the standard deviation of 50 configurations over 10 ps. The seemingly small changes in Fig. 6a are nevertheless responsible for a significant volume increase, because ICO clusters are so much more densely packed than RD clusters. Our 
Voronoi analysis shows that the total volume increase is $3.3 \%$ and that nearly one third (30\%) of this can be attributed to ICO to RD cluster transformations (Fig. 6b).

\section{Discussion}

The distinctive atomic motion detected in the present work, and depicted in Fig. 2a, fits the term "rattling" rather better than other modes. The rattling is identifiable as the 'fast processes' in Fig. 1, with a high frequency of mechanism $(40 \mathrm{GHz})$ that is essentially independent of temperature. The rattling atoms are congregated in loosely packed zones (Fig. 5a) that resemble those proposed as the sites of the different relaxation modes in metallic glasses [11-13,15]. String-like motion (Fig. 3) that permits the rattling has also been suggested to be the fundamental event underlying $\beta$ relaxation [29], and has been used to explain the link between $\beta$ relaxation and the diffusion of small atoms in metallic glasses [4]. Yet, in these studies the string-like motions are clearly thermally activated, unlike the rattling. The rattling, involving some string-like coordination of the atomic movements, is thus most easily interpreted as a small-amplitude (and possibly shorter-string) precursor to fast $\beta$ ' and later to $\beta$ relaxation.

The spatial distribution of rattling atoms in the undeformed glass (Fig. 5a) resembles that of the fraction of atoms participating in soft modes in $\mathrm{Cu}_{64} \mathrm{Zr}_{36}$ glass, as found by Ding et al. [17] (Fig. 4 in their paper). In each case, the correlation length of the soft spots is $\sim 1 \mathrm{~nm}$. Ding et al. [17] show that the sites of shear transformation zones (STZs, the mediators of plastic flow in metallic glasses [30]) are strongly correlated with GUMs and their associated soft spots. The spacing between STZs found by Ding et al. [17] is $\sim 2.5 \mathrm{~nm}$, indeed similar to that between clusters of rattling atoms in the present work (Fig. 5a). 
We conclude that the loosely packed regions, where the rattling is concentrated, are important in initiating plastic flow at STZs. We can expect that, initially at least, the response of the glass would be dominated by the fast processes in these regions, rather than by other mass transport events that are more energetically costly. The link between plastic flow and rattling is emphasized by the strong increase in frequency of occurrence of the rattling, and in the number of rattling atoms associated with soft spots, when the glass is subjected to a tensile strain of $10 \%$ (Fig. $5 b$ ).

The presence of loosely packed regions in the glass could be attributed to insufficient relaxation. But incomplete relaxation is intrinsic to glasses. Greater degrees of relaxation would reduce the population of loosely packed regions, and reduce the frequency of occurrence of rattling motions, but would not eliminate them.

\section{Conclusions}

Analysis of atomic coordinates in an MD-simulated $\mathrm{Cu}_{65} \mathrm{Zr}_{35}$ glass at $300 \mathrm{~K}$ shows that a small portion of the system's atoms perform rattling motions between two neighboring sites. The to-and-fro motions show a wide distribution of distances and lifetimes, and appear distinct from the motions attributed to the boson peak. The rattling events take place with string-like concerted motion of two or three neighboring atoms and are accompanied by local changes in the atomic coordination, some of which are persistent. The frequency of the rattling mechanism is $40 \mathrm{GHz}$, independent of temperature. With these characteristics, we identify the observed rattling between sites as the mechanism (for our simulated metallic 
glass) of the fast processes that are characteristic of the glassy state in general and that lie in the frequency range between $\beta$-relaxation and the boson peak.

The rattling atoms are the centers predominantly of geometrically imperfect coordination polyhedra; these are inhomogeneously distributed, concentrated in relatively low-density regions that have a wide range of size. In the quiescent glass (not subjected to externally imposed strain) at $300 \mathrm{~K}$, the frequency of occurrence of rattling events (normalized by the total number of atoms in the system) is $430 \mathrm{MHz}$ and rises with temperature. The frequency at $300 \mathrm{~K}$ rises to $630 \mathrm{MHz}$ when the glass is subjected to a tensile strain of $10 \%$. The strain leads to more atoms performing rattling motions, congregated in larger loosely packed regions. Changes in atomic coordination polyhedra, of the kind associated with rattling motions, account for one third of the total volume increase resulting from the imposed strain. The predominant structural change in the glass is a reduction in the relative proportion of icosahedral coordination polyhedra.

The wide distributions of relaxation times associated with $\alpha, \beta$ and $\beta$ ' relaxation modes ensure that there are regions of the frequency (or temperature) spectrum where the different modes overlap and are coupled. The observed rattling of atoms in a concerted string-like motion shows that these fast processes have a link with $\beta$ relaxation, suggesting that the processes (which, in contrast to the relaxation itself, show little or no thermal activation) are a low-amplitude, short-string precursor. These precursor fast processes appear to be the origin of the shear transformation zones (STZs) that ultimately trigger plastic flow. They are concentrated in loosely packed regions, the distribution and extent of which are important in determining the mechanical properties of metallic glasses. 


\section{Acknowledgements}

PPR's work is supported by VitriMetTech Project FP7-PEOPLE-2013-ITN-\#607080. ALG acknowledges support from the European Research Council under the European Union's Horizon 2020 research and innovation programme (grant ERC-2015-AdG-695487:

ExtendGlass). The authors thank C. M. Meylan for useful discussions and for assistance in preparation of the manuscript.

\section{References}

[1] P. Lunkenheimer, U. Schneider, R. Brand, A. Loidl, Glassy dynamics, Contemp. Phys. 41 (2000) 15-36.

[2] H.B. Yu, W.H. Wang, H.Y. Bai, K. Samwer, The $\beta$-relaxation in metallic glasses, Nation. Sci. Rev. 1 (2014) 429-461.

[3] P. Lunkenheimer, A. Loidl, Dielectric spectroscopy of glass-forming materials: $\alpha$ relaxation and excess wing, Chem. Phys. 284 (2002) 205-219.

[4] H.B. Yu, K. Samwer, Y. Wu, W.H. Wang, Correlation between $\beta$ relaxation and selfdiffusion of the smallest constituting atoms in metallic glasses, Phys. Rev. Lett. 109 (2012) 095508.

[5] H.B. Yu, W.H. Wang, K. Samwer, The $\beta$ relaxation in metallic glasses: an overview, Mater. Today 16 (2013) 183-191.

[6] J.C. Qiao, Y.-J. Wang, L.Z. Zhao, L.H. Dai, D. Crespo, J.M. Pelletier, L.M. Keer, Y. Yao, Transition from stress-driven to thermally activated stress relaxation in metallic glasses, Phys. Rev. B 94 (2016) 104203. 
[7] A.P. Sokolov, E. Rössler, A. Kisliuk, D. Quitmann, Dynamics of strong and fragile glass formers: Differences and correlation with low-temperature properties, Phys. Rev. Lett. 71 (1993) 2062-2065.

[8] J.C. Qiao, J.M. Pelletier, Dynamic mechanical analysis in La-based bulk metallic glasses: secondary $\beta$ and main $\alpha$ relaxations, J. Appl. Phys. 112 (2012) 083528.

[9] H.B. Yu, K. Samwer W.H. Wang, H.Y. Bai, Chemical influence on $\beta$-relaxations and the formation of molecule-like metallic glasses, Nature Comm. 4 (2013) 2204.

[10] Z. Lu, B.S. Shang, Y.T. Sun, Z.G. Zhu, P.F. Guan, W.H. Wang, H.Y. Bai, Revealing $\beta$-relaxation mechanism based on energy distribution of flow units in metallic glass, J. Chem. Phys. 144 (2016) 144501.

[11] Q. Wang, S.T. Zhang, Y. Yang, Y.D. Dong, C.T. Liu, J. Lu, Unusual fast secondary relaxation in metallic glass, Nature Comm. 6 (2015) 7876.

[12] L.Z. Zhao, R.J. Xue, Z.G. Zhu, K.L. Ngai, W.H. Wang, H.Y. Bai, A fast dynamic mode in rare earth based glasses, J. Chem. Phys. 144 (2016) 204507.

[13] H.Y. Jiang, P. Luo, P. Wen, H.Y. Bai, W.H. Wang, M.X. Pan, The near constant loss dynamic mode in metallic glass, J. Appl. Phys. 120 (2016) 145106.

[14] Y. Li, H.Y. Bai, W.H. Wang, K. Samwer, Low-temperature specific-heat anomalies associated with the boson peak in CuZr-based bulk metallic glasses, Phys. Rev. B 74 (2006) 052201.

[15] B. Huang, Z.G. Zhu, T.P. Ge, H.Y. Bai, B.A. Sun, Y. Yang, C.T. Liu, W.H. Wang, Hand in hand evolution of boson heat capacity anomaly and slow $\beta$-relaxation in Labased metallic glasses, Acta Mater. 110 (2016) 73-83.

[16] N. Jakse, A. Nassour, A. Pasturel, Structural and dynamic origin of the boson peak in a Cu-Zr metallic glass, Phys. Rev. B 85 (2012) 174201. 
[17] J. Ding, S. Patinet, M.L. Falk, Y.-Q. Cheng, E. Ma, Soft spots and their structural signature in a metallic glass, PNAS 111 (2014) 14052-14056.

[18] V.N. Novikov, Vibration anharmonicity and fast relaxation in the region of the glass transition, Phys. Rev. B 58 (1998) 8367-8378.

[19] G.A. Almyras, Ch.E. Lekka, N. Mattern, G.A. Evangelakis, On the microstructure of the $\mathrm{Cu}_{65} \mathrm{Zr}_{35}$ and $\mathrm{Cu}_{35} \mathrm{Zr}_{65}$ metallic glasses, Scripta Mater. 62 (2010) 33-36.

[20] M.F. de Oliveira, G.A. Almyras, G.A. Evangelakis, Structural differences of amorphous $\mathrm{Cu}_{65} \mathrm{Zr}_{35}$ between rapidly quenched and topologically destabilized crystalline $\mathrm{Cu}$ and $\mathrm{Zr}$ metals by molecular dynamics simulations, Comput. Mater. Sci. 104 (2015) 92-97.

[21] Y.Q. Cheng, H.W. Sheng, E. Ma, Relationship between structure, dynamics, and mechanical properties in metallic glass-forming alloys, Phys. Rev. B 78 (2008) 014207.

[22] S. Nosé, A molecular dynamics method for simulations in the canonical ensemble, Molecular Phys. 52 (1984) 255-268.

[23] H.C. Andersen, Molecular dynamics simulations at constant pressure and/or temperature, J. Chem. Phys. 72 (1980) 2384-2393.

[24] L. Tayebi, Ch.E. Lekka, G.A. Evangelakis, Poisson ratio under compressive and tensile strain; effect on the mechanical response of the $\mathrm{Cu}_{46} \mathrm{Zr}_{54}$ metallic glass, Phys. Stat. Sol. A 205 (2008) 2603-2606.

[25] D.G. Papageorgiou, I.N. Demetropoulos, I.E. Lagaris, MERLIN-3.1.1. A new version of the Merlin optimization environment, Comput. Phys. Commun. 159 (2004) 70-71.

[26] C.H. Rycroft, Voro++: A three-dimensional Voronoi cell library in C++, Chaos 19 (2009) 041111. 
[27] J. Ding, Y.-Q. Cheng, E. Ma, Full icosahedra dominate local order in $\mathrm{Cu}_{64} \mathrm{Zr}_{34}$ metallic glass and supercooled liquid, Acta Mater. 69 (2014) 343-354.

[28] Ch.E. Lekka, A. Ibenskas, A.R. Yavari, G.A. Evangelakis, Tensile deformation accommodation in microscopic metallic glasses via subnanocluster reconstructions, Appl. Phys. Lett. 91 (2007) 214103.

[29] D. Bedorf, K. Samwer, Length scale effects on relaxations in metallic glasses, J. NonCryst. Solids 356 (2010) 340-343.

[30] M. Zink, K. Samwer, W.L. Johnson, S.G. Mayr, Plastic deformation of metallic glasses: size of shear transformation zones from molecular dynamics simulations, Phys. Rev. B 73 (2006) 172203. 


\section{Figure Captions}

Fig. 1. Schematic spectrum of frequency-dependent dielectric loss in glass-forming systems, showing characteristic dynamic modes, in particular $\alpha$ and $\beta$ relaxation. Reproduced with permission from Lunkenheimer and Loidl, Chem. Phys. 284 (2002) 205-219. Copyright 2015 Elsevier Science B.V.

Fig. 2. (a) The time evolution of a representative atom's coordinates $x, y$ and $z$, demonstrating rattling motion at $300 \mathrm{~K}$. The bars at the top indicate the character of the atomic coordination polyhedra: icosahedral (ICO), rhombic dodecahedral (RD) and cuboctahedral (CUB). (b) Close-up view of the rattling motion shown in (a).

Fig. 3. Ball-and-spoke representation of a particular rattling event. In this region of the $\mathrm{Cu}_{65} \mathrm{Zr}_{35}$ glass, four states separated by tens of ps, are represented through three inter-state plots: a (from State 1 to State 2), b (from 2 to 3), and c (from 3 to 4). White spheres represent atomic positions 'before' (i.e. State 1 in plot a) and atoms that do not belong to the neighborhood of the central atoms $\mathrm{A}$ and $\mathrm{B}$. Colored spheres ( $\mathrm{Cu}$ in orange and $\mathrm{Zr}$ in blue) represent atomic positions 'after' (i.e. State 2 in plot a). Lower color saturation indicates depth. Black arrows indicate the movement direction. (a) From State 1 to State 2: A and B are the central atoms of two face-sharing clusters. Atoms B, D, A and C participate in a rattling movement of about $1 \AA$, involving a string-like sequence. (b) From State 2 to State 3: neighboring atoms readjust their positions through small movements of the order of 0.3 
$\AA$ and atom B comes back to its initial position. (c) From State 3 to State 4: a different string-like sequence of movements can be identified. Atoms A and B are now first neighbors, and the coordinated rattling movements have changed the local atomistic structure.

Fig. 4. Atomic volume, from Voronoi tessellation, around the atoms performing rattling motions (RM) and around the rest of the atoms in the system (green). The distributions have been normalized to have unit area. The solid black lines are fitted Gaussians. (a) for $\mathrm{Cu}$, the rattling atoms show an average atomic volume $1 \%$ higher than that for the others. (b) for $\mathrm{Zr}$, the rattling atoms show an average atomic volume $1.5 \%$ higher than of the others. The shoulder on the low-volume side of the distribution for rattling $\mathrm{Zr}$ atoms is not explained.

Fig. 5. Representative distribution of the rattling atoms $(\mathrm{Cu}$ in orange and $\mathrm{Zr}$ in blue) of the atoms in the simulated glass. The cubic box represents the simulation boundaries and weaker color indicates that the atoms are closer to the back of the box. Only small fractions of the atoms in the system perform rattling movements: (a) $1.1 \%$ in the quiescent glass (with no external load), and (b) $2 \%$ when the glass is subjected to a tensile strain of $10 \%$. The rattling atoms are inhomogeneously distributed in loosely packed regions that are larger in the strained glass.

Fig. 6. The evolution of glass structure under tensile strain. (a) The relative proportions of types of clusters (central atom and nearest-neighbor shell). The clusters are characterized as 
coordination polyhedra and may take distorted forms: icosahedral (ICO), rhombic dodecahedral (RD) and cuboctahedral (CUB). The absolute shifts in the proportions, as a result of the strain, are: ICO 1.8\% decrease; RD 1.2\% increase; and CUB $0.6 \%$ increase. (b) Volume evolution: at an imposed tensile strain of $10 \%$, the volume increase directly associated with the transformation of ICO clusters to RD and CUB types accounts for nearly one third $(30 \%)$ of the total volume increase of the glassy system. Calculated errors are smaller than the points' size.

\section{Graphical abstract}

The time evolution of a representative atom's coordinates $x, y$ and $z$, demonstrating rattling motion at $300 \mathrm{~K}$. 


\section{FIGURES}

Figure 1:

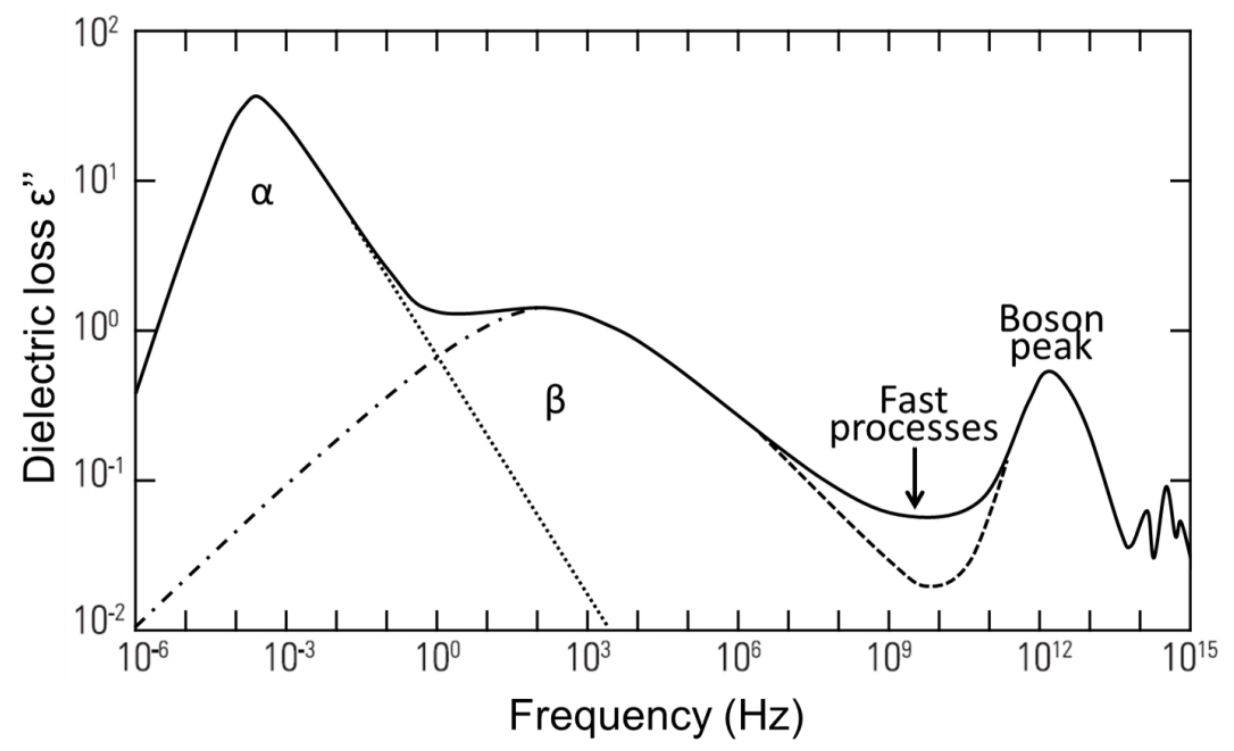


Figure 2:
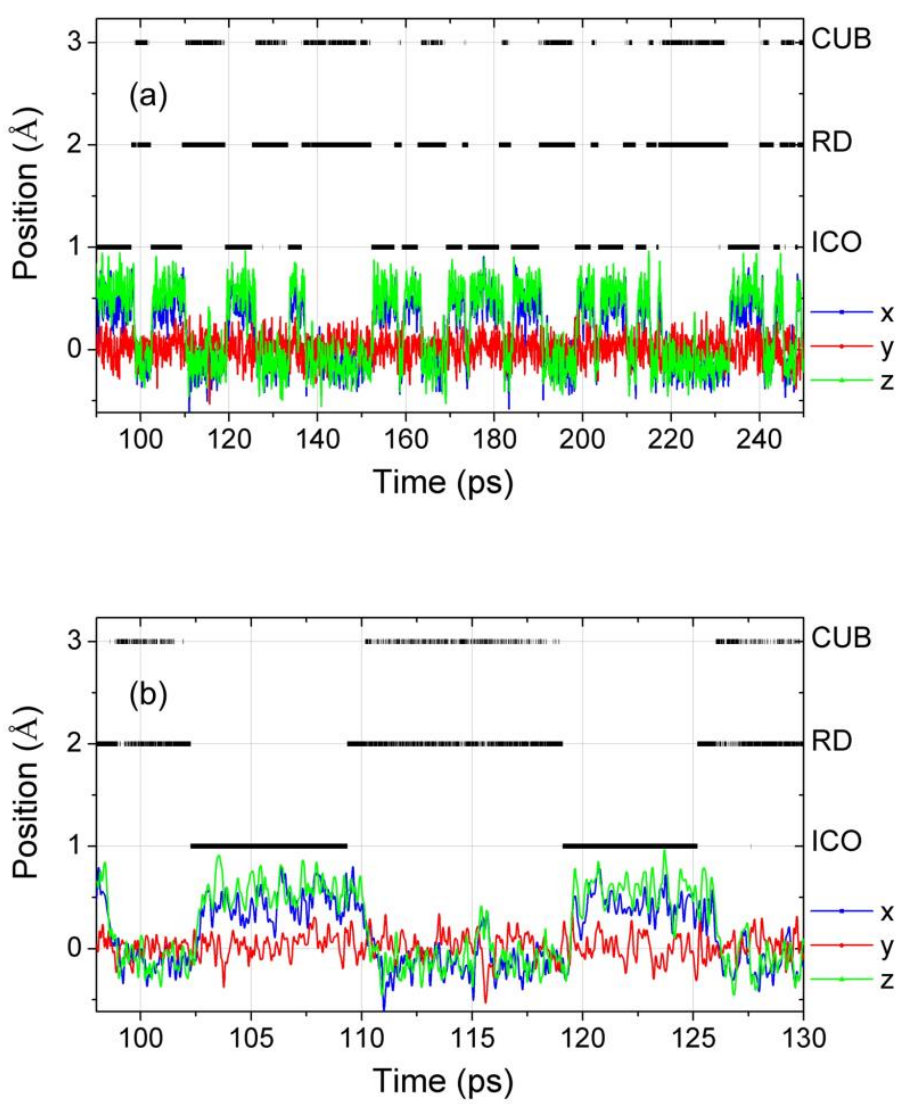
Figure 3:

(a) State $1 \rightarrow$ State 2 .

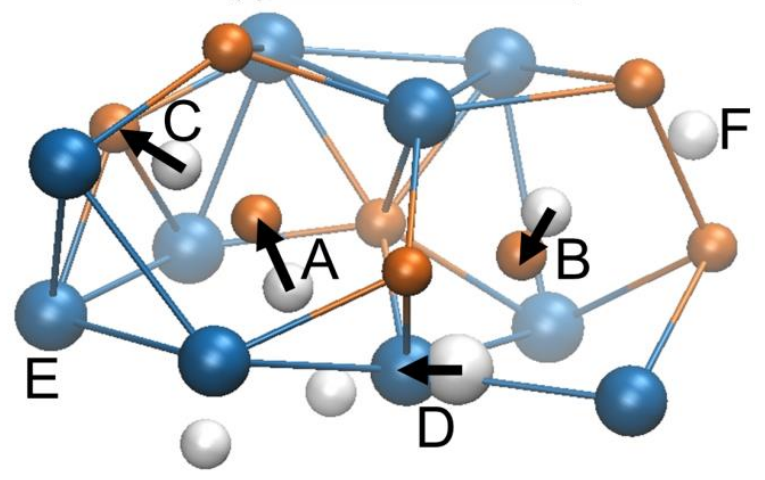

(b) State $2 \rightarrow$ State 3 .

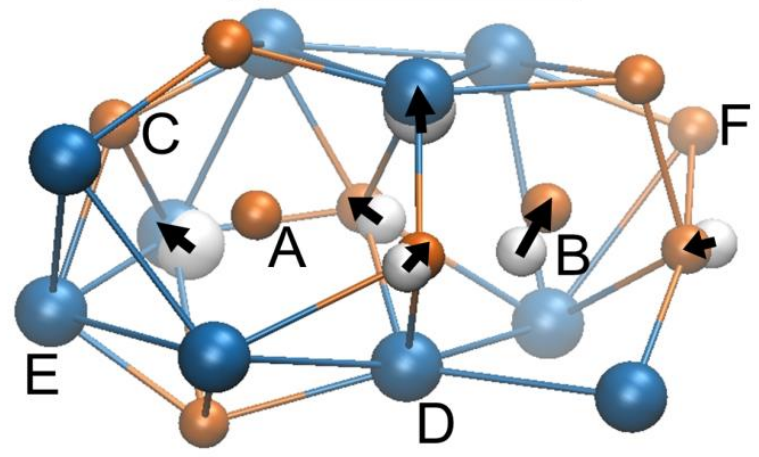

(c) State $3 \rightarrow$ State 4 .

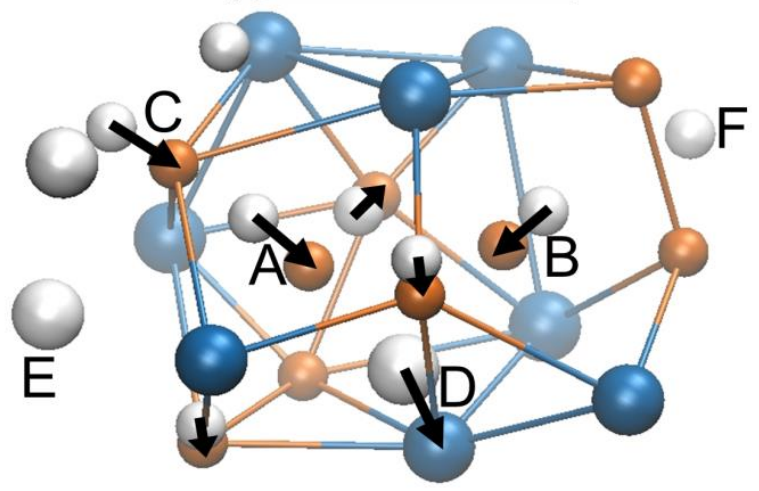


Figure 4:
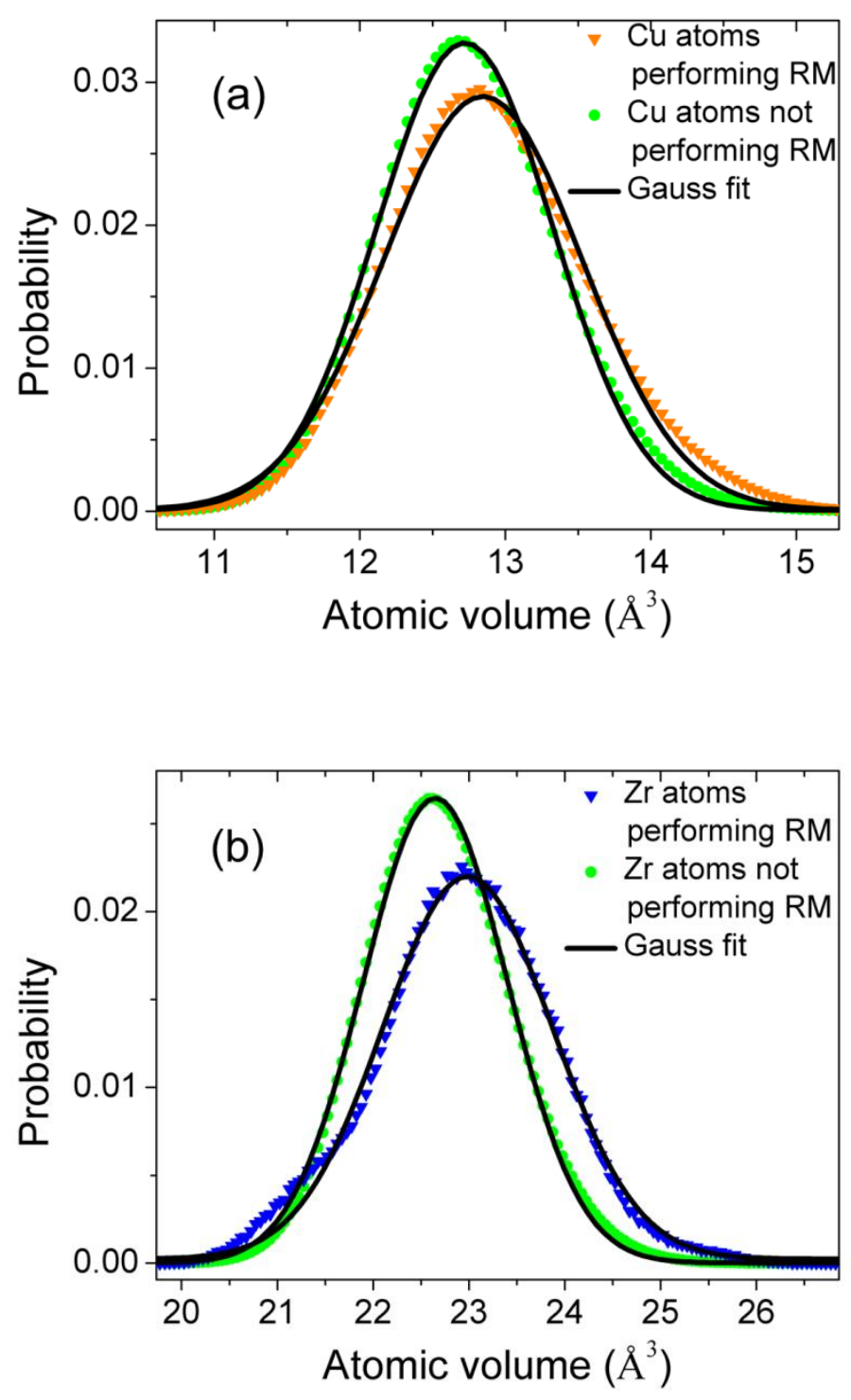
Figure 5:

(a) Quiescent

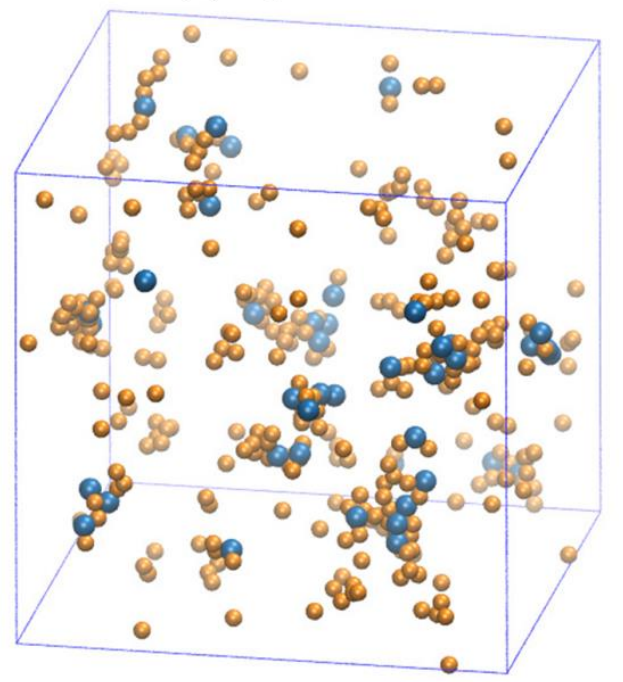

(b) Under strain

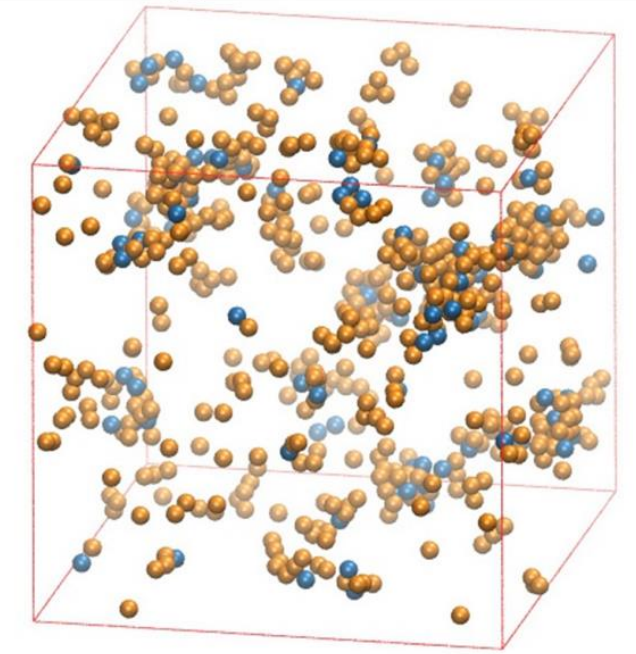


Figure 6:
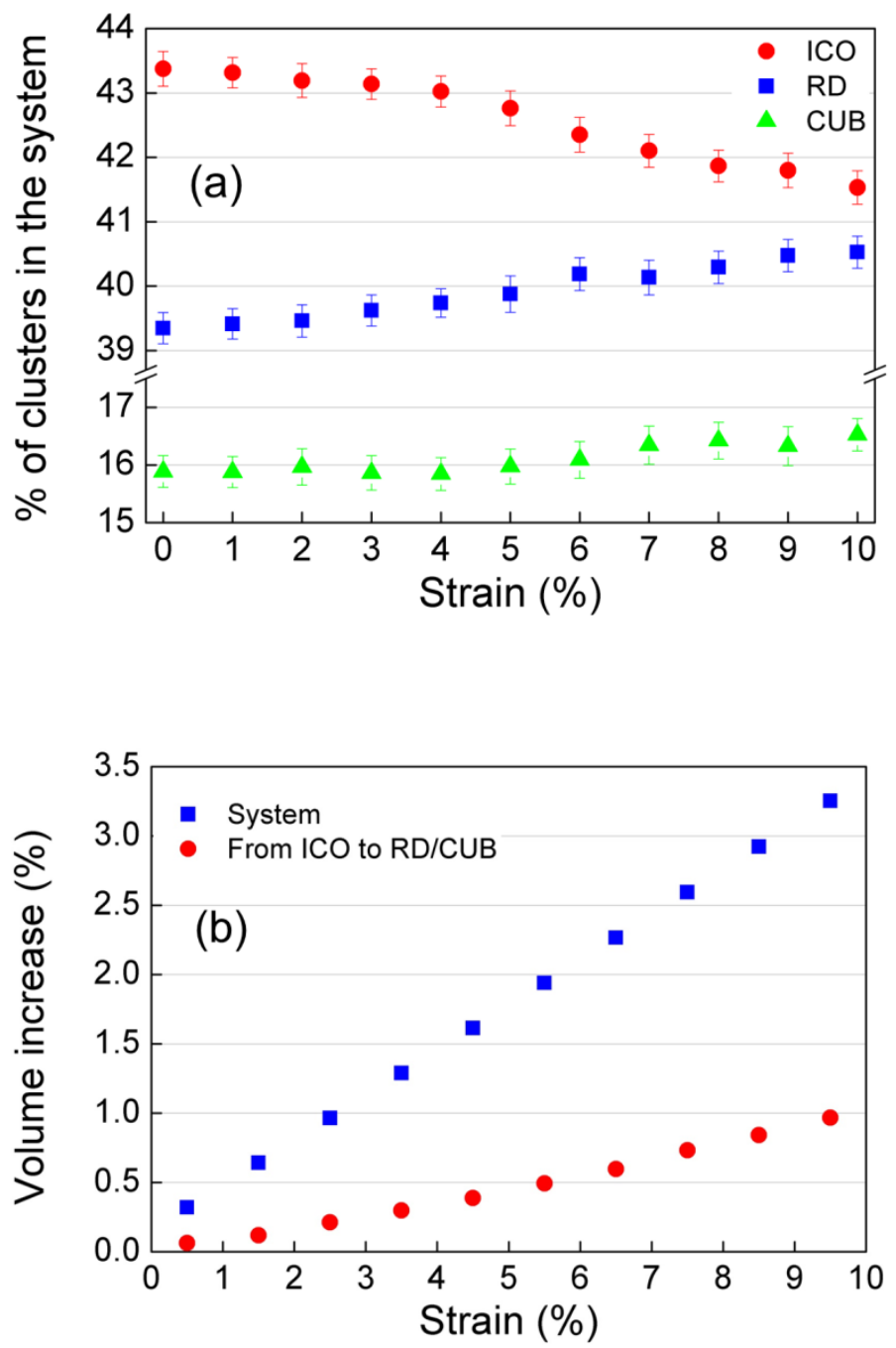